\title{
Molecular Characterization of Mycobacterium kansasii Isolates in the State of São Paulo between 1995-1998
}

\author{
Erica Chimara+ , Carmen Maria Saraiva Giampaglia, Maria Conceição Martins, \\ Maria Alice da Silva Telles, Suely Yoko Mizuka U eki, Lucilaine Ferrazoli
}

Setor de Micobactérias, Seção de Bacteriologia, Instituto Adolfo Lutz, São Paulo, Av. Dr. Arnaldo 355, 01246-902, SP, Brasil

Mycobacterium kansasii is the most common cause of pulmonary nontuberculous mycobacteria infection and classical identification of this pathogen needs a time consuming phenotypic tests. Polymerase chain reactionrestriction fragment lenght polymorphism analysis (PRA) of the gene enconding for the 65kDa heat shock (hsp65) protein offers an easy, rapid, and inexpensive procedure to identify and subtype $\mathrm{M}$. kansasii isolates. In the present study, we performed a retrospective analysis of patients who had mycobacteria identified on the basis of phenotypic tests by means of a review of database at Mycobacteria Laboratory of the Instituto Adolfo Lutz in the period 19951998. A total of 9381 clinical isolates were analyzed of which 7777 (82.9\%) were identified as $\mathrm{M}$. tuberculosis complex and 1604 (17.1\%) as nontuberculous mycobacteria. Of the 296 M. kansasii isolates, 189 (63.8\%) isolates obtained from 119 patients were viable and were analyzed by PRA-hsp65. Hundred eight two (98.9\%) were classified as M. kansasii type I. Two isolates were classified as type II and III and five isolates were characterized as other Mycobacterium species. Clinical isolates of M. kansasii in the state of São Paulo was almost exclusively subtype I regardless of HIV status.

Key words: Mycobacterium kansasii - nontuberculous mycobacteria - hsp65 - restriction analysis

Mycobacterium kansasii is an acid-fast photochromogenic bacterium, which has been identified as a disease agent worldwide. This pathogen has been frequently isolated from tap water, showers or distribution networks, and can be acquired from the environment rather than human-to-human transmission (Collins et al. 1984, Falkinham 1996). M. kansasii commonly causes pulmonary infection, similar to pulmonary tuberculosis, with highest incidence in men over 50, who suffer from chronic obstructive pulmonary disease (Martinez-Moragon et al. 2001, Koh et al. 2002).

The incidence of disease caused by M. kansasii varies according to geographic region. Studies carried out in the United States of America and Canada reported a higher frequency of mycobacteria of the $M$. avium complex (MAC), whereas M. kansasii was more frequently found in England and Spain (Martínez-Moragón et al. 2001). Before the acquired immune deficiency syndrome (AIDS) epidemic, $M$. kansasii was the agent responsible for pulmonary infections in patients with previous pulmonary disease, but the disseminated disease was extremely rare. Currently, M. kansasii is the second species of nontuberculous mycobacteria (NTM) that causes disseminated disease in HIV-positive patients (Bloch et al. 1998). Disseminated M. kansasii infection in HIV-positive patients establishes the diagnosis of the AIDS (Brasil 2003).

Until the end of the 1980s, identification of M. kansasii was achieved based on phenotypic characteristics only (Collins et al. 1997). However, the phenotypic tests for

Financial support: Capes

+Corresponding author.Fax: +55-11-30668179. E-mail: echimara@ial.sp.gov.br

Received 16 June 2004

Accepted 4 October 2004 identification are technically demanding and time-consuming. Two to four weeks of incubation are needed to obtain the results (Lebrun et al. 1992). Although the treatment of the symptomatic infection by mycobacteria is indicated upon positive result in the microscopic observation of the clinical specimen, the rapid identification of the infecting species can contribute to more effective treatment (Plikaytis et al. 1992).

At the beginning of the 1990s, the first test commercially available for genotypic identification of $M$. kansasii was the Accuprobe test (Gen-Probe, San Diego, CA), based on the hybridization of a DNA sequence, labeled with a chemoluminescent substance, with the ribosomal RNA of grown mycobacteria (Knott et al. 1997). The use of these and other specific DNA sequences for $M$. kansasii, such as probes for the identification of isolates obtained from different geographic areas, revealed genetic variability of this species (Huang et al. 1991, Ross et al. 1992, Yang et al. 1993).

In 1992, Plikaytis et al. developed a method for mycobacteria identification that combines amplification by the polymerase chain reaction (PCR) in a DNA sequence highly conserved among Mycobacterium genus and the restriction enzyme analysis of the amplified sequence. This DNA sequence corresponds to a portion (1380 pb) of the gene that codes for the $h s p 65$ heat shock protein. Analysis of the restriction profile of the amplified sequence from the genome of $30 \mathrm{M}$. kansasii isolates showed the existence of five restriction patterns. Telenti et al. (1993), using primers for amplification of the $h s p 65$ gene with $439 \mathrm{pb}$ and other restriction enzymes (PRA-hsp65), were able to differentiate $M$. kansasii from $M$. gastri that presented a similar sequence for the 16SrRNA gene. Devallois et al. (1997) applied this method and described patterns for 34 species and established five different types for $M$. kansasii. 
The aim of the present study was to characterize $M$. kansasii isolates by analyzing the patterns of the PRA$h s p 65$. This characterization was performed to confirm the species previously determined by conventional tests, to determine the prevalence of different genetic types in São Paulo and to compare the genetic types of clinical isolates obtained from HIV-positive and negative patients.

\section{MATERIALS AND METHODS}

Patient identification - Retrospective analysis of patients who had mycobacteria identified on the basis of conventional tests was carried out by means of a review of database at Mycobacteria Laboratory of the Instituto Adolfo Lutz in the period 1995-1998. From this database, informations were obtained about species identification, date of species identification, site of isolation, and HIV status. The isolates identified as $M$. kansasii were selected, subcultured on Löwenstein-Jensen (L-J) medium and analyzed by PRA- $h s p 65$.

Mycobacteria identification - The clinical isolates were initially screened for distinction of $M$. tuberculosis complex from NTM by direct observation of colony aspect and presence/absence of cord formation on ZiehlNeelsen stained smears. Cord-positive isolates were further subcultured in L-J medium containing $0.5 \mathrm{mg} / \mathrm{ml}$ of p-nitrobenzoic acid and $5 \mu \mathrm{g} / \mathrm{ml}$ of 2-thiophene-carboxylic acid hydrazide to confirm $M$. tuberculosis complex identification. NTM were identified to species level using tests based on phenotypic characteristics, such as growth rate, pigment production, growth in different temperatures $(25$, 37 , and $45^{\circ} \mathrm{C}$ ), growth in the presence of specific agents (antibiotics, sodium salicylate), and biochemical tests (nitrate reduction, catalase activity, urease activity, tween 80 hydrolysis) (Tsukamura 1984, Collins et al. 1997).

Genomic DNA extraction of M. kansasii isolates - A loop of mycobacteria growth on L-J medium was suspended in $500 \mu \mathrm{l}$ of sterile water, boiled for $10 \mathrm{~min}$ and frozen at $-20^{\circ} \mathrm{C}$ for at least $18 \mathrm{~h}$.

PRA-hsp65 - The amplification of the $439 \mathrm{pb}$ fragment from the $h s p 65$ gene was performed using primers $\mathrm{Tb} 11$ (5'-ACCAACGATGGTGTGTCCAT) and Tb12 (5'CTTGTCGAACCGCATACCCT), described by Telenti et al. (1993). For the amplification reaction, a mixture was made containing $50 \mathrm{mM} \mathrm{KCl}, 10 \mathrm{mM}$ Tris- $\mathrm{HCl}, 1.5 \mathrm{mM}$ $\mathrm{MgCl}_{2}, 10 \%$ glycerol, $0.2 \mathrm{mM}$ of each deoxynucleotide, $0.5 \mu \mathrm{M}$ of each primer (Tb11 and Tb12), $1 \mathrm{U}$ of Taq polymerase and $5 \mu \mathrm{l}$ of lysed bacteria. The mixture was submit- ted to 45 amplification cycles $\left(1 \mathrm{~min}\right.$ at $94^{\circ} \mathrm{C}, 1 \mathrm{~min}$ at $60^{\circ} \mathrm{C}$, and $1 \mathrm{~min}$ at $72^{\circ} \mathrm{C}$ ), followed by $10 \mathrm{~min}$ of extension at $72^{\circ} \mathrm{C}$. The amplicons were digested with BstEII and HaeIII enzymes, separately. Digested fragments were separated by electrophoresis in agarose at $3 \%$ in TBE $0.5 \mathrm{x}$ buffer and were analyzed visually. Restriction patterns were analyzed using the algorithm described by Devallois et al. (1997) and also by PRASITE query (http://app.chuv.ch/ prasite/index.html).

\section{RESULTS}

Patient identification - From January 1995 to December 1998, 9381 isolates were analyzed at Instituto Adolfo Lutz on the basis of conventional tests. A total of 7777 $(82.9 \%)$ were identified as $M$. tuberculosis complex and $1604(17.1 \%)$ as NTM (Table I). Of the 296 M. kansasii isolates, $189(63.8 \%)$ isolates from 119 patients were viable and were analyzed by PRA- $h s p 65$. Of them, 42 (35.3\%) patients had more than one isolate analyzed by PRAhsp 65 . The 189 isolates were cultured from sputum (157), bronchial washes (6), pus (5), blood (4), skin biopsy (3), abscesses (3), bone marrow (2), urine (2), gastric aspirate (2), synovial fluid (2), pericardic fluid (1), cerebrospinal fluid (1), and pleural fluid (1). Of the 119 patients, 36 (30.2\%) patients had at least three $M$. kansasii isolated from nonsterile clinical specimens or one from sterile ones, 22 (18.5\%) patients had two M. kansasii isolated from sputum and $59(49.6 \%)$ patients had a single isolate from nonsterile specimen.

PRA-hsp65 - The analysis of the restriction patterns of the $h s p 65$ gene from 189 isolates detected three of the five $M$. kansasii genetic types described in the literature. Of the 189 isolates, $182(98.9 \%)$ presented the type I pattern, one presented type II and one type III (Table II). Type II and III strains were isolated from HIV-positive patients (Table II). Five isolates were identified as other species by genetic characterization (M. tuberculosis complex, $M$. gordonae, $M$. fortuitum, $M$. simiae, $M$. sp.) (Table II). Among them, two (M. simiae, M. fortuitum) were obtained from patients with other isolates identified as $M$. kansasii. M. fortuitum, was isolated from sputum of one patient with other two M. kansasii isolated from sputum. M. simiae isolate was obtained from blood of a HIV positive patient two years later the isolation of $M$. kansasii from the sputum. Genetic patterns detected by the PRAhsp 65 method for the isolates studied can be seen in the Figure.
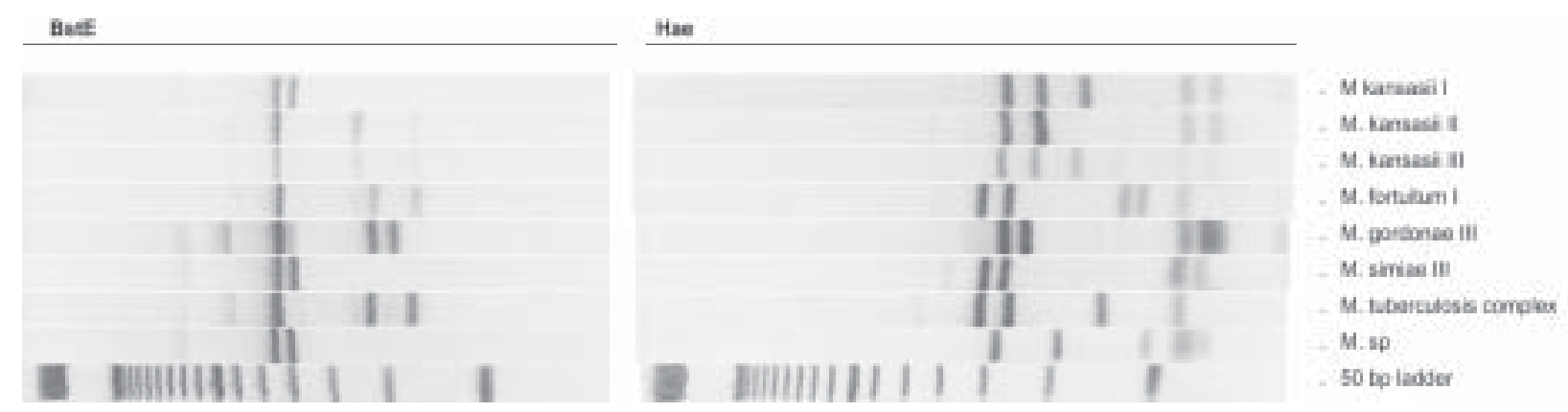

Different genetic patterns from 189 clinical isolates analyzed by restriction patterns of $h s p 65$ gene, $M$. $s p$ : restriction patterns of $h s p 65$ gene pattern not described in the literature. 
TABLE I

Clinical isolates identified on the basis of phenotypic tests at the Mycobacteria Laboratory of the Instituto Adolfo Lutz between 1995-1998

\begin{tabular}{lrr}
\hline Species & $\mathrm{N}$ & $(\%)$ \\
\hline M. tuberculosis & 7775 & 82.88 \\
M. bovis - BCG & 2 & 0.02 \\
\hline M. avium complex & 936 & 9.98 \\
M. kansasii & 296 & 3.16 \\
M. gordonae & 113 & 1.20 \\
M. chelonae & 77 & 0.82 \\
M. fortuitum & 66 & 0.70 \\
M. terrae complex & 19 & 0.20 \\
M. xenopi & 9 & 0.10 \\
M. marinum & 5 & 0.05 \\
M. genavense & 2 & 0.02 \\
M. szulgai & 2 & 0.02 \\
M. celatum & 1 & 0.01 \\
M. sp ${ }^{\text {a }}$ & 70 & 0.75 \\
Mix culture & 8 & 0.09 \\
\hline Total & 9381 & 100.00 \\
\hline
\end{tabular}

$a$ : species confirmed by $h s p 65$ sequencing only; $b$ : inconclusive result by conventional tests

\section{DISCUSSION}

Mycobacteria Laboratory of the Instituto Adolfo Lutz is a reference laboratory and receives clinical isolates from all the state of São Paulo for identification and susceptibility tests. During the period of the study, M. tuberculosis was the most often species identified, (82.9\%) followed by MAC (9.9\%) and M. kansasii (3.1\%). These two species of NTM were the most frequently reported in Brazil and in another places around the world (MartínezMoragón et al. 2001, da Silva Rocha et al. 2002). It is important to note that NTM frequently occur as transient commensals in the upper respiratory tract, intestine, and skin. Also, they frequently contaminate clinical specimens. Because of this, guidelines for determining the clinical significance of a positive NTM isolates were published (American Thoracic Society 1997, British Thoracic Society 2000). These guidelines suggest that isolation of NTM on multiple occasions or on a single occasion from a ster- ile site should be supported by evidence of an actual disease process (Choudhri et al. 1995, American Thoracic Society 1997). Although this was not the scope of this study, we note that the number of NTM cultures received by the reference laboratory exceeds the number of the patients with disease. In this study, only $30.2 \%$ of the patients had more than three M. kansasii isolated from non-sterile site or one from sterile one. Once laboratory do not have clinical information, we could not establish how many of these patients had in fact disease caused by NTM.

The restriction enzyme analysis of the hsp 65 gene, amplified from the genome of isolates previously identified as $M$. kansasii by phenotypic tests, confirmed that the vast majority $(97.3 \%)$ of the isolates examined do belong, in fact, to this species. In addition to being a fast test, the PRA method is relatively simple and its use in many laboratories resulted in the construction, in 1999, of the Internet site (PRASITE) http://app.chuv.ch/prasite/ index.html. Smole et al. (2002) proved the efficiency of PRA method while studying MAC isolates. According to Smole et al. (2002) and Silva et al. (2001), the PRA method is faster than conventional methods, cheaper than commercial probes and theoretically applicable to all mycobacteria species.

Discordant results were obtained for five isolates only. Discordant results found could be due to the presence of more than one mycobacterial species in the original culture and when subcultured just one species was recovered. Another possible explanation is that identification of NTM using phenotypic methods could result in incorrect classification due to the complexity of the parameters involved in performing and reading the tests.

In the present study, three (I, II, and III) of the five genetic types of $M$. kansasii described by Alcaide et al. (1997) were detected, and the subtype I was the most frequent $(98.9 \%)$. This result is similar to that reported by da Silva Rocha et al. (2002) that used the same method to characterize $20 \mathrm{M}$. kansasii clinical isolates collected from 16 different states of Brazil. The three genetic types were also the only ones detected among the 163 clinical isolates studied by Alcaide et al. (1997), 111 (68\%) of them were characterized as type I, $50(31 \%)$ type II, and only 2 $(1 \%)$ as type III. Of the 113 isolates obtained from the

TABLE II

Molecular characterization by PRA-hsp65 method of isolates phenotypically identified as Mycobacterium kansasii

\begin{tabular}{|c|c|c|c|c|c|c|}
\hline \multirow[b]{3}{*}{ Species } & \multirow{3}{*}{$\begin{array}{l}\text { Genetic } \\
\text { type }\end{array}$} & \multirow{3}{*}{$\begin{array}{c}\text { Number of } \\
\text { isolates } \\
(\mathrm{n}=189)\end{array}$} & \multicolumn{4}{|c|}{ Number of patients } \\
\hline & & & \multirow{2}{*}{$\begin{array}{c}\text { Total } \\
(\mathrm{n}=119)\end{array}$} & \multicolumn{3}{|c|}{ HIV status ${ }^{a}$} \\
\hline & & & & $\mathrm{P}$ & $\mathrm{N}$ & I \\
\hline M. kansasii & I & $182(96.29)$ & $112(94.12)$ & 44 & 5 & 63 \\
\hline M. kansasii & II & $1(0.53)$ & $1(0.84)$ & 1 & - & - \\
\hline M. kansasii & III & $1(0.53)$ & $1(0.84)$ & 1 & - & - \\
\hline M. fortuitum & I & $1(0.53)$ & $1(0.84)$ & 1 & - & - \\
\hline M. gordonae & V & $1(0.53)$ & $1(0.84)$ & 1 & - & - \\
\hline M. simiae & II & $1(0.53)$ & $1(0.84)$ & 1 & - & - \\
\hline M. tuberculosis & - & $1(0.53)$ & $1(0.84)$ & 1 & - & - \\
\hline M. $s p^{b}$ & - & $1(0.53)$ & $1(0.84)$ & - & - & 1 \\
\hline
\end{tabular}

a: HIV status, P: positive, N: negative, I: unknown; $b$ : PRA-hsp65 pattern not described in the literature. 
environment, Alcaide et al. (1997) characterized $18(16 \%)$ as type II, $32(28 \%)$ as type III, $60(53 \%)$ as type IV, and 3 (3\%) as type V. A recent American study (Zhang et al. 2004), found similar results when recovered 78 of 81 (96.3\%) clinical isolates with indistinguishable PRA pattern which matched PRA type I.

Unlike Alcaide et al. (1997), Picardeau et al. (1997) detected type I among the clinical isolates (42\%) as well as among isolates from water (32\%), 8 of the 16 clinical isolates were isolated from HIV-positive patients. Similarly, Alcaide et al. (1997) identified the other genetic types, as often in clinical isolates as in isolates from water. Eigth of 13 clinical isolates type II were isolated from HIV-positive patients. It is important to emphasize that in the present study, the only isolate of $M$. kansasii characterized as type II was isolated from an HIV-positive patient.

Among clinical isolates worldwide, type I is the predominant type of M. kansasii in all studies to date. The prevalence of genetic type I among the isolates of $M$. kansasii of human origin detected in the studies performed by Alcaide et al. (1997), Picardeau et al. (1997), and Zhang et al. (2004), as in the present study, indicate that this type of strain may present an association of virulence confering upon it a greater capacity for colonization and/or pathogenic activity for human beings, while the data available for the strains of genetic type II suggest that is a type less able to overcome natural resistance mechanisms and behaving in a more opportunistic manner.

Many epidemiological and pathogenic aspects are still far from being understood. Those first studies using molecular methods have already demonstrated the complexity of the problem by describing the heterogeneous nature of the strains. Clinical isolates of $M$. kansasii in the state of São Paulo was almost exclusively subtype I regardless of HIV status. The demonstration that this type is more frequent in clinical specimens than the other types, which are found in environmental samples, question the environmental origin of the pathogenic strains, even though there has been no demonstration of person-toperson transmission (Garay et al. 2001). The application of these molecular techniques in future epidemiological studies seem essential to addressing issues raised about habitat and the transmission mechanisms of M. kansasii.

\section{REFERENCES}

Alcaide F, Richter I, Bernasconi C, Springer B, Hagenau C, Schulze-Röbbcke R, Tortoli E, Martin R, Böttger EC, Telenti A 1997. Heterogeneity and clonality among isolates of Mycobacterium kansasii: implications for epidemiological and pathogenicity studies. J Clin Microbiol 35: 19591964.

American Thoracic Society 1997. Diagnosis and treatment of disease caused by nontuberculous mycobacteria. Am Rev Respir Dis 156: S1-S25.

Bloch KC, Zwerling L, Pletcher MJ, Hahn JA, Gerbeling JL, Ostroff SM, Vugia DJ, Reingold AL 1998. Incidence and clinical implications of isolation of Mycobacterium kansasii: results of a 5-year, population-based study. Ann Intern Med 129: 698-704.

Brasil, Ministério da Saúde 2003. Critérios de definição de casos de aids em adultos e crianças, Série manuais nr 60, 56 pp.
British Thoracic Society 2000. Management of opportunistic mycobacterial infections: Joint Tuberculosis Committee Guidelines 1999. Thorax 55: 210-218.

Choudhri S, Manfreda J, Wolfe J, Parker S, Long R 1995. Clinical significance of nontuberculous mycobacteria isolates in a Canadian tertiary care center. Clin Infect Dis 21: 128-133.

Collins CH, Grange JM, Yates MD 1984. A review: mycobacteria in water. J Appl Bacteriol 57: 193-211.

Collins CH, Grange JM, Yates MD 1997. Tuberculosis Bacteriology. Organization and Practice, 2nd ed., Butterworth Heinemann, Oxford.

da Silva Rocha A, Werneck Barreto AM, Dias Campos CE, Villas-Boas da Silva M, Fonseca L, Saad MH, Degrave WM, Suffys PN 2002. J Clin Microbiol 40: 4191-4196.

Devallois A, Goh KS, Rastogi N 1997. Rapid identification of mycobacteria to species level by PCR-restriction fragment length polymorphism analysis of the $h s p 65$ gene and proposition of an algorithm to differentiate 34 mycobacterial species. J Clin Microbiol 35: 2969-2973.

Falkinham JO 1996. Epidemiology of infection by nontuberculous mycobacteria. Clin Microbiol Rev 9: 177-215.

Garay JG, Cebrian FG, Saco GM, Blasco JJL, de Gordejuela ER 2001. Enfermedad pulmonary por Mycobacterium kansasii. Análisis de 39 casos. Arch Bronconeumol 37: 27 34.

Huang ZH, Ross BC, Dwyer B 1991. Identification of Mycobacterium kansasii by DNA hybridization. J Clin Microbiol 29: 2125-2129.

Knott C, Bott M, Yamagata S, Riggan D, Brentano S, Andruszkiewicz I, Endozo T, Mathews G 1997. Evaluation of the reformulated Gen-Probe Mycobacterium kansasii Accuprobe test for the identification of M. kansasii from culture, 97th General Meeting of the American Society for Microbiology, Miami Beach, Florida, Abstract C-328.

Lebrun L, Espinasse F, Poveda JD, Levy-Frebault VV 1992. Evaluation of nonradioactive DNA probes for identification of mycobacteria. J Clin Microbiol 30: 2476-2478.

Koh WJ, Kwon OJ, Lee KS 2002. Nontuberculous mycobacterial pulmonary diseases in immunocompetent patients. Korean J Radiol 3: 145-157.

Martínez-Moragón E, Menéndez R, Palasí P, Santos M, López Aldeguer J 2001. Enfermedades por micobactérias ambientales en pacientes con y sin infección por el VIH: características epidemiológicas, clínicas y curso evolutivo. Arch Bronconeumol 37: 281-286.

Picardeau M, Prod'hom G, Rskine L, Lepennec MP, Vincent V 1997. Genotypic characterization of five subspecies of Mycobacterium kansasii. J Clin Microbiol 35: 25-32.

Plikaytis BB, Plikaytis BD, Yakrus MA, Butler WR, Woodley CL, Silcox VA, Shinnick TM 1992. Differentiation of slowly growing Mycobacterium species including Mycobacterium tuberculosis, by gene amplification and restriction fragment lenght polymorphism analysis. J Clin Microbiol 30: 18151822.

Ross BC, Jackson K, Yang M, Sievers A, Dwyer B 1992. Identification of a genetically distinct subspecies of Mycobacterium kansasii. J Clin Microbiol 30: 2930-2933.

Silva CF, Ueki SYM, Geiger DCP, Leão SC 2001. hsp-65 PCRrestriction enzyme analysis (PRA) for identification of mycobacteria in the clinical laboratory. Rev Inst Med Trop São Paulo 43: 25-28.

Smole SC, McAleese F, Ngampasutadol J, Forfham von Reyn C, Arbeit RD 2002. Clinical and epidemiological correlates of genotypes within the Mycobacterium avium Complex defined by restriction and sequence analysis of hsp65.J Clin Microbiol 40: 3374-3380.

Telenti A, Marchesi F, Balz M, Bally F, Böttger EC, Bodmer T 
1993. Rapid identification of mycobacteria to the species level by polymerase chain reaction and restriction enzyme analysis. J Clin Microbiol 31: 175-178.

Tsukamura M 1984. Identification of Mycobacteria, Obu, Aichi: The National Chubu Hospital, Japan.

Yang M, Ross BC, Dwyer B 1993. Isolation of a DNA probe for identification of Mycobacterium kansasii, including the genetic subgroup. J Clin Microbiol 31: 2769-2772.

Zhang Y, Mann LB, Wilson RW, Brown-Elliott BA, Vincent V, Iinuma Y, Wallace Jr RJ 2004. Molecular analysis of Mycobacterium kansasii isolates from the United States. J Clin Microbiol 42: 119-125. 
